\title{
Energy balance in apodized diffractive multifocal intraocular lenses
}

\author{
Francisco Alba-Bueno ${ }^{* a}$, Fidel Vega ${ }^{\mathrm{a}}$, María S. Millán ${ }^{\mathrm{a}}$ \\ ${ }^{a}$ Optics and Optometry Department, Polytechnic University of Catalonia.
}

\begin{abstract}
The energy distribution between the distance and near images formed in a model eye by three different apodized diffractive multifocal intraocular lenses (IOLs) is experimentally determined in an optical bench. The model eye has an artificial cornea with positive spherical aberration (SA) similar to human cornea. The level of SA upon the IOL, which is pupil size dependent, is controlled using a Hartmann-Shack wave sensor. The energy of the distance and near images as a function of the pupil size is experimentally obtained from image analysis. All three IOLs have the same base refractive power (20D) but different designs (aspheric, spherical) and add powers $(+4.0 \mathrm{D},+3.0 \mathrm{D})$. The results show that in all the cases, the energy efficiency of the distance image decreases for large pupils, in contrast with the theoretical and simulated results that only consider the diffractive profile of the lens. As for the near image, since the diffractive zone responsible for the formation of this image has the same apodization factor in the spherical and aspheric lenses and the apertures involved are small (and so the level of SA), the results turn out to be similar for all the three IOL designs.
\end{abstract}

Keywords: intraocular lens, multifocal lens, diffractive lens, imaging efficiency

\section{INTRODUCTION}

The crystalline lens in the eye provides the ability of focusing objects at different distances in a process called accommodation. This lens grows throughout life and increases in size and rigidity, causing the loss of the accommodation capacity (presbyopia) around the 45-50 years and later the loss of transparency (cataracts). Cataract is the most common cause of visual impairment in the world [1]; the surgical treatment of cataract involves the extraction and replacement of the crystalline by an intraocular lens (IOL).

Nowadays the implantation of diffractive multifocal intraocular lenses (DMIOLs) is a common procedure intended to avoid the spectacle dependence in near vision. The DMIOLs uses the base lens curvature and the zero $(m=0)$ and first $(m=1)$ diffraction orders to achieve two focal points (often referred as to optical powers) that corresponds to the far and near foci respectively [2], [3]. These designs allow the pseudophakic eye to correctly focus at different distances but have an inherent drawback: the focused retinal image formed by one of the powers of the DMIOLs is always overlaid by an out of focus image from the other lens power. This effect may lead to visually disturbing phenomena such as halos and/or glare perceptions [4] depending on the illumination conditions, the axial distance between the two images and their relative energy distribution. For these reasons, the contrast sensitivity in eyes implanted with DMIOLs may be worse than those implanted with monofocal IOLs [5]. To fully understand the nature of these effects it is interesting to characterize in a test bench [6] the optical performance of these IOLs. The Point Spread Function and Modulation Transfer Function are metrics widely used in other works to characterize the optical quality of different IOLs [7][8]. It is less common, however, to analyze the energy distribution between the distance and near images and its variation with the pupil diameter. The energy distribution is an optical quality feature that is especially important in the case of the apodized diffractive multifocal intraocular lenses (ADMIOLs) like the AcrySof ${ }^{\circledR}$ ResTOR $^{\circledR}$ (Alcon, Fort Worth, Texas, USA), which are specifically designed with a twofold purpose: to reduce the glare and halo phenomena and, in addition to this, to have an increasing distance-dominant behavior for large pupil sizes. The latter implies to make the energy distribution between the distance and near images dependent on the eye pupil. Furthermore, some of these IOLs have aspheric surfaces, and there is a great interest to determine in a test bench [9][10] and in clinical studies [11][12][13] the advantages of this design versus the spherical one, particularly when some studies have shown little or no benefit of aspheric IOLs operating with small pupils [13][14].

In this work the energy balance between distance and near images of ADMIOLs of different design (aspheric, spherical) and add powers $(+4.0 \mathrm{D},+3.0 \mathrm{D})$, is experimentally obtained as a function of the pupil size in a test bench. The

*francisco.alba-bueno@upc.edu Tel 937398678 Fax 937398301

22nd Congress of the International Commission for Optics: Light for the Development of the World, edited by Ramón Rodríguez-Vera, Rufino Díaz-Uribe, Proc. of SPIE Vol. 8011, 80119G

2011 SPIE · CCC code: $0277-786$ X/11/\$18 $\cdot$ doi: $10.1117 / 12.902895$

Proc. of SPIE Vol. 8011 80119G-1 
experimental results are compared to the theoretical predictions that only consider the diffractive profile of the lens. We aim to study the performance of these ADMIOLs in a model eye that induces SA values upon the IOL similar to those naturally induced by a human eye. The final goal is to determine whether the aspheric design or a particular add power would really improve the IOL performance.

\section{METHOD}

\subsection{Intraocular lens description and theoretical energy balance}

The ADMIOLs AcrySof ${ }^{\circledR}$ ResTOR ${ }^{\circledR}$ are described in detail in Refs. [15][16]. These lenses have an optical zone of $6 \mathrm{~mm}$ diameter with a hybrid diffractive-refractive design on its anterior surface (figure 1, left). The diffractive region covers the central $3.6 \mathrm{~mm}$ of the lens and diverts light simultaneously into the zero $(m=0)$ and first $(m=1)$ diffraction orders that correspond to the distance and near foci, respectively. The outer region of the IOL is purely refractive and sends light to the distance focus exclusively. The anterior base surface of the lens may be either spherical or aspheric, the latter being intended to introduce negative SA and thus, to partially compensate for the natural positive SA of human corneas [17][18]. This compensation, expressed by means of the c[4,0] Zernike coefficient is $0.20 \mu \mathrm{m}$ for a $6 \mathrm{~mm}$ eye pupil [19].

The radii of the diffractive rings (and thus the number of diffractive zones) are determined by the design wavelength and the add power [20]:

$$
r_{i}^{2}=(2 i-1) \lambda \frac{1000}{D_{\text {add }}}
$$

where $i$ is the zone number, $\lambda$ is the design wavelength, and $D_{\text {add }}$ is the add power (in Diopters). Thus, for $\lambda=550 \mathrm{~nm}$ and $D_{\text {add }}=+3.0 \mathrm{D}$, the radius of the central disk is $r_{l}=0.428 \mathrm{~mm}$ whereas it reduces to $r_{l}=0,371 \mathrm{~mm}$ for $D_{\text {add }}=+4,0 \mathrm{D}$.

The height $h_{i}$ of the diffractive steps smoothes towards the periphery (apodizing profile in figure 1, right). An apodization factor is given by Lee et al:

$$
f_{i}^{\text {apodized }}=1-\left(\frac{r_{i}}{r_{\text {outer }}}\right)^{2} \text { for } r_{i}=0, r_{1}, r_{2}, \ldots, r_{\text {outer }},
$$

for which the step height reduction is given by:

$$
h_{i}=f^{\text {apodized }} \cdot h_{0},
$$

where $h_{0}$ is the maximum height at the optical axis $(r=0)$. The diffractive profile of the lens acts as an optical interface between the IOL material (refractive index $n_{I O L}=1.55$ ) and the aqueous medium (refractive index $n_{\text {aqueous }}=1.336$ ) where the lens is immersed. Because of the refractive index difference $n_{I O L}-n_{\text {aqueous }}$, the light waves passing through different parts of the diffractive profile are phase shifted by different amount. The maximum induced phase shift (in wavelengths units) is:

$$
\alpha_{i}=\left(n_{\text {IOL }}-n_{\text {aqueous }}\right)^{h_{i}} / \lambda
$$

with $h_{i}$ calculated with Equation (3).
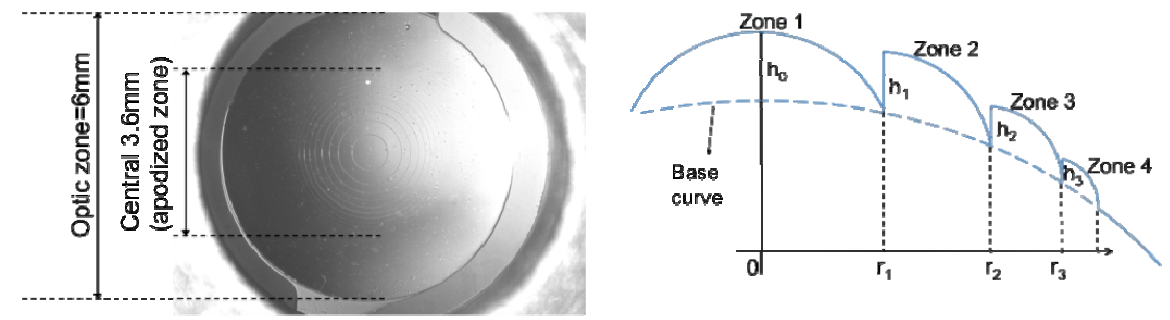

Figure 1. Left: Image and dimensions of one of the measured ADMIOLs. Right: Sketch of the apodization of the central zone. 
By setting $h_{0}=1.3 \mu \mathrm{m}$ [16] in Equation (4) the value of $\alpha_{i}$ at the first diffractive zone is $\alpha_{0}=0.51$, which is close to half wave. The apodization of the diffractive profile of the MDIOLs implies that the value of $\alpha_{i}$ progressively decreases from the center to the periphery of the diffractive zone, which has important implications on how the light is distributed between the $m=0$ (distance power) and $m=1$ (near power) diffraction orders as a function of the pupil aperture or, equivalently, as a function of the number of diffractive rings that are illuminated.

If $\alpha_{i}$ were constant for all the rings, the diffraction throughput efficiency (TE) of the $m=0$ and $m=1$ orders would be given by [21]:

$$
T E_{m=0,1}=\operatorname{sinc}^{2}\left(m-\alpha_{i}\right) .
$$

However, since the value of $\alpha_{i}$ varies with the radius, the $T E$ for each $\alpha_{i}\left(T E_{m=0,1}^{i}\right)$ has to be weighted by a factor that corresponds to the ith-diffractive ring area. Therefore, the energy that the diffractive part of the IOL would divert from an incident plane wave into the $m=0$ and $m=1$ diffraction orders is calculated by means of linear combinations of the weighted contributions of the rings:

$$
\begin{aligned}
& I_{m=0}^{\text {diffractive }}=c_{t e} \cdot \sum_{i=1}^{n} A^{i} \cdot T E_{m=0}^{i}, \\
& I_{m=1}^{\text {diffractive }}=c_{t e} \cdot \sum_{i=1}^{n} A^{i} \cdot T E_{m=1}^{i},
\end{aligned}
$$

where $c_{t e}$ is a proportionality constant and $n$ is the number of diffractive rings of area $A^{i}$ that are illuminated and thus are taking part on the diffraction process.

With a reduced pupil aperture for which the IOL only operates with the first diffractive zone (i.e., $\left.\alpha_{i}=\alpha_{0}\right)$ there is nearly equal diffraction throughput efficiencies for the distance $\left(T E_{m=0}^{0}=0.38\right)$ and near $\left(T E_{m=1}^{0}=0.43\right)$ powers. With larger pupils, more diffractive rings are illuminated but the progressive reduction of the phase shift of the waves $<$ as they pass through the outer diffractive rings implies that $T E_{m=0}^{i}>T E_{m=1}^{i}$, and according to Equations (6) and (7) the energy sent to the distance power $(m=0)$ becomes reinforced at the expenses of the near power $(m=1)$.

In the case of the purely refractive region of the ADMIOL, the light goes exclusively to the distance power i.e. $T E_{m=0}^{\text {refractive }}=1$, and therefore the energy is simply:

$$
I_{m=0}^{\text {refractive }}=c_{t e} \cdot A^{\text {refractive }} \cdot T E_{m=0}^{\text {refractive }},
$$

where $A^{\text {refractive }}$ is the area of the illuminated refractive region of the ADMIOL.

Then, the amount of energy sent to either the distance and near powers strongly depend on the size of the pupil or, equivalently, on the size of the illuminated area of the IOL (referred from now on as to IOL-pupil) and can be calculated as:

$$
\begin{aligned}
& I_{m=0}=I_{m=0}^{\text {diffractive }}+I_{m=0}^{\text {refractive }}, \\
& I_{m=1}=I_{m=1}^{\text {diffractive }},
\end{aligned}
$$

which can be expressed in terms of energy efficiency as:

$$
\begin{aligned}
& \frac{I_{m=0}}{I_{I O L}^{\text {total }}}, \\
& \frac{I_{m=1}}{I_{\text {IOL }}^{\text {total }}},
\end{aligned}
$$

where $I_{\text {IOL }}^{\text {tot }}$ is the total energy transmitted through the whole IOL aperture. This energy is proportional to the area of the IOL aperture $A_{I O L}$ provided that any loss of energy (for instance caused by scattering in the diffractive steps) [22] is neglected:

$$
I_{I O L}^{\text {total }}=c_{\text {te }} \cdot A_{I O L}
$$


We have calculated the energy efficiencies according to Equations (11) and (12), as a function of the IOL-pupil diameter in the case of the AcrySof ${ }^{\mathbb{B}} \operatorname{ReSTOR}^{\circledR}$. The results, plotted in Fig. 2, are in excellent agreement with those reported elsewhere [15][16] and show that there is a different energy balance between the two optical powers depending on the pupil size. Thus, for small pupils the energy is nearly equally divided between the two foci whereas for large pupils the energy balance changes in favor of the distance focus.

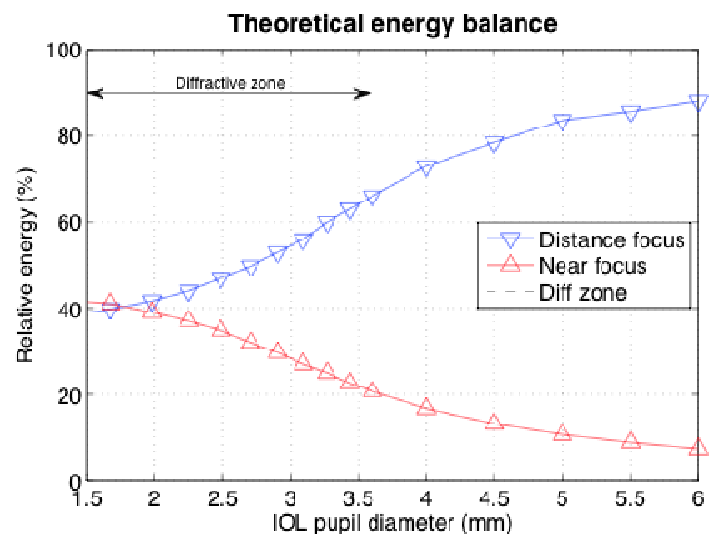

Figure 2. Theoretical energy efficiency of the distance and near powers in an ADMIOL of +4.0D of addition.

However, it must be emphasized that these calculations do not take aberrations into account. They only predict the amount of the energy sent to either the distance or near powers for a particular IOL pupil, but they do not ensure that this energy will be properly focused onto the respective images, the latter depending on several factors like the design of the base lens (either aspheric or spherical) where the diffractive profile is made, or the level of SA upon the IOL (which turns out to depend on the type of the cornea and the pupil size). This fact is especially relevant when the ADMIOL is inserted in a model eye with an aberrated cornea [23][24] the latter meaning that there would be a converging beam impinging on the IOL with a value of SA that depends on the size of the pupil aperture.

The ADMIOLs used in this study are the three Acrysof ${ }^{\circledR}$ ReSTOR ${ }^{\circledR}$ available in the market: SN60D3, SN6AD3 and SN6AD1. Their characteristics are shown in Table 1. Their monofocal counterparts with both spherical (SN60AT) and aspheric (SN60WF) designs will be also considered for the sake of comparison.

Table 1. Characteristics of the IOLs studied.

\begin{tabular}{|llllll|}
\hline & SN60AT & SN60WF & SN60D3 & SN6AD3 & SN6AD1 \\
\hline Type & Monofocal & Monofocal & Bifocal & Bifocal & Bifocal \\
First surface & Spherical & Aspherical & Spherical - & Aspherical - & Aspherical - \\
& & & Apodized - & Apodized - & Apodized - \\
& & & Diffractive & Diffractive & Diffractive \\
Second surface & Spherical & Spherical & Spherical & Spherical & Spherical \\
Base power $(D)$ & $20.0 \mathrm{D}$ & $20.0 \mathrm{D}$ & $20.0 \mathrm{D}$ & $20.0 \mathrm{D}$ & $20.0 \mathrm{D}$ \\
Addition $(D)$ & - & - & +4.0 & +4.0 & +3.0 \\
& & & & & \\
\hline
\end{tabular}

\subsection{Optical bench}

To assess the real energy distribution between distance and near images formed by ADMIOLs, we have designed the optical bench sketched in Figure 3 [6]. The IOL is inserted in a model eye formed by an iris diaphragm (that acts as the Entrance Pupil-(EP)) the artificial cornea and the wet cell. The model eye is in accordance with the ISO standard requirements [25] except for the artificial cornea. 
Instead of using an aberration free artificial cornea as the ISO standard recommends, we use an equiconvex spherical lens that induces an amount of SA at the IOL plane similar to that naturally induced by the human cornea [17][18][26]. It is important to realize that the size of the EP determines on one hand the IOL-pupil and, on the other hand, the level of SA produced by the artificial cornea upon the IOL.

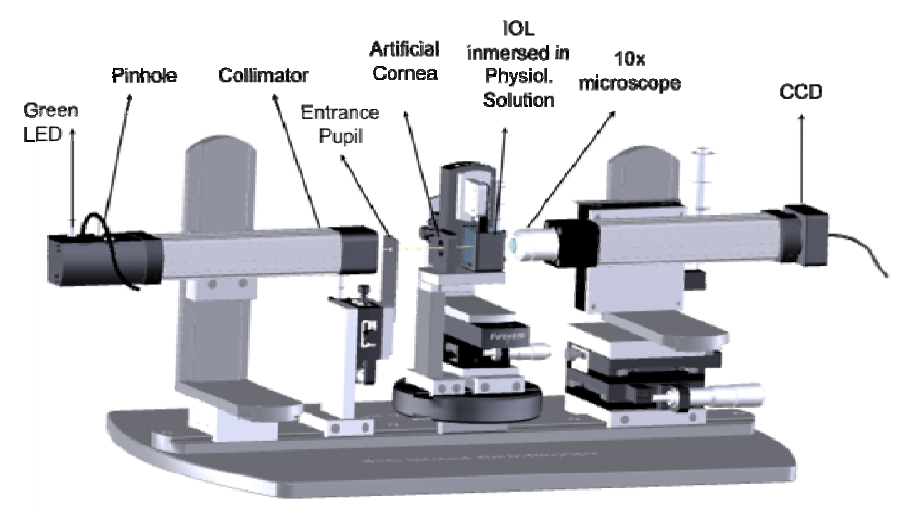

Figure 3. Optical setup to implement a model eye and test IOLs. A pinhole object at infinity is imaged by the DMIOL into the distance and near images that, in turn, can be sequentially focused on the CCD sensor.

We have simulated the dependence of the SA of the wavefront that leaves the artificial cornea and impinges on the IOL as a function of the IOL-pupil using commercial optical design software (Zemax Development Corporation, San Diego, US). This simulation has been experimentally verified using a Hartmann-Shack wavefront sensor and it is shown in Fig. 4.

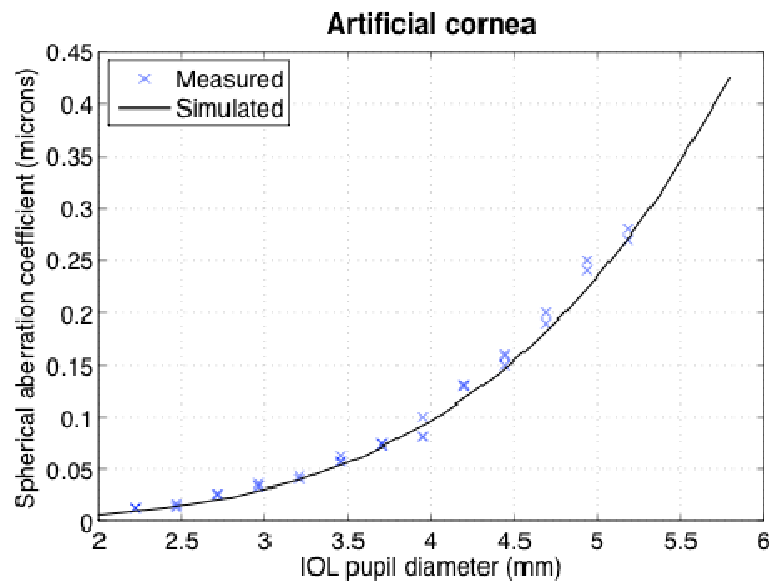

Figure 4. Black line: Calculated value of the Zernike $\mathrm{c}[4,0] \mathrm{SA}$ coefficient as a function of the IOL-pupil diameter of the artificial cornea (fused silica; $n=1.46 R_{l}=39.53 \mathrm{~mm} ; R_{2}=-39.53 \mathrm{~mm} ; e=4.9 \mathrm{~mm}$ ). Blue crosses: measured value of the Zernike c[4,0].

\subsection{Energy balance from image analysis.}

The energy distribution between the distance and near images as a function of the EP diameter is obtained through image analysis as shown in Fig. 3. The quasi-monochromatic LED with a narrowband emission centered at $\lambda=521 \mathrm{~nm}$ illuminates the $200 \mu \mathrm{m}$ pinhole object. A collimator produces a collimated beam that illuminates the model eye (artificial cornea plus wet cell) where the IOL is inserted. When an ADMIOL is tested, the pinhole object is imaged in two planes separated along the optical axis. These images are referred to as near and distance images, respectively. An infinite corrected microscope mounted in a high precision translation holder is used to select either the distance or the near image and magnify it onto an 8-bit image acquisition CCD camera. 
Examples of the near and distance images of the pinhole object obtained with an ADMIOL AcrySof RESTOR are shown in Fig. 5(a) and Fig. 5(b), respectively. Both images basically consist of the focused image of the pinhole (labeled $I_{\text {pinh }}$ in Fig. 5) surrounded by blurred background or halo (labeled $I_{\text {backg }}$ in Fig 5). This background corresponds primarily to the overlaying defocused image. In the case of the distance image (Fig. 5(b)), however, there is an additional contribution that strongly depends on level of SA upon the IOL as it will be shown in the next paragraphs. The energy of the image just in the focused pinhole region $\left(I_{p i n h}\right)$, and the energy of the total image that comprises the pinhole plus background regions $\left(I_{\text {total }}=I_{\text {pinh }}+I_{\text {backg }}\right)$, are obtained by integration of the pixel grey level in the corresponding regions:

$$
I_{R}=\sum_{n \text { pixel }}^{n \in R} g(n)
$$

where $R$ stands for either the pinhole region or the total image $(R=$ pinh, total $), n$ is a pixel contained in the $R$ region, and $g(n)$ is the pixel grey level. Since the images are blurred because of the background, it is not straightforward to determine the borders of the region that corresponds just to the focused pinhole. An edge detection algorithm (Sobel edge gradient detector, [27]) was used to remove all the background contribution outside the pinhole (Figures 5(c) and 5(d)). Then, $I_{p i n h}$ is calculated according to Equation (14) from these filtered images.
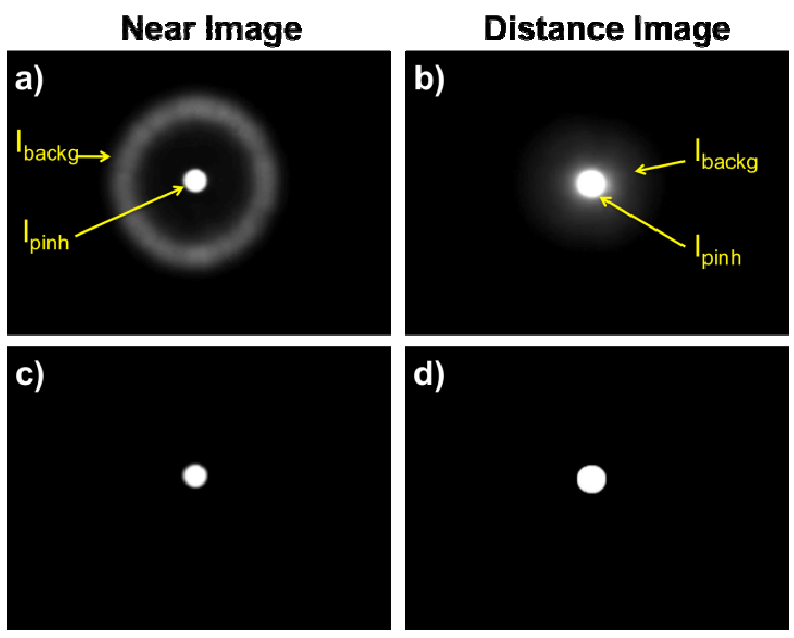

Figure 5. a) and b): Near and distance images experimentally obtained with an ADMIOL in the model eye. The arrows point to the region named pinhole (pinh) and background (backg) respectively. c) and d): same as above after removing the background.

\section{RESULTS AND DISCUSSION}

In order to establish the role of the SA upon the IOL and how the lens design (aspheric vs. spherical) may help to tackle it, we have started studying the variation of the energies $I_{\text {total }}$ and $I_{\text {pinh }}$ obtained with the monofocal spherical (SN60AT) and aspheric (SN60WF) IOLs. The results are plotted, as a function of the IOL-pupil diameter in Fig. 6. The corresponding relative energies, defined as $I_{\text {pinh }} / I_{\text {total }}$, are plotted in Fig. 7. To make the comparison of results easier, $I_{\text {total }}$ and $I_{\text {pinh }}$ are normalized to the value of $I_{\text {total }}$ obtained with the largest pupil diameter $(6 \mathrm{~mm})$. The results in Fig. 6 show that for both types of IOLs and small IOL-pupil diameters up to $3 \mathrm{~mm}$ (when the level of SA upon the IOL is small), the energy $I_{\text {pinh }}$ is nearly the same as $I_{\text {total }}$, which means that the background contribution is negligible in these conditions, no matter the type (spherical or aspheric) of monofocal IOL is. As a consequence, images of high relative energy of the order of $80 \%$ are obtained with small apertures for both IOLs as it is shown in Fig. 7.

For larger EP diameters a different behavior between the spherical and aspheric IOLs can be observed. In the case of the spherical SN60AT, the energy $I_{\text {pinh }}$ remains constant, even though the measured $I_{\text {total }}$ increases (figure 6 , left). This result implies that, when opening the entrance pupil, most of the additional available energy is not sent to the pinhole image but 'wasted' in the background, and consequently, a dramatic reduction of the image efficiency occurs (see Fig. 7, left). For 
the aspheric SN60WF, the larger the entrance pupil the larger the value of $I_{\text {pinh }}$, but the increase occurs with a slope smaller than the increase of $I_{\text {total }}$ (Fig. 6, right), which implies a moderate reduction of its relative energy for larger EP diameters as it is shown in Fig. 7, right.

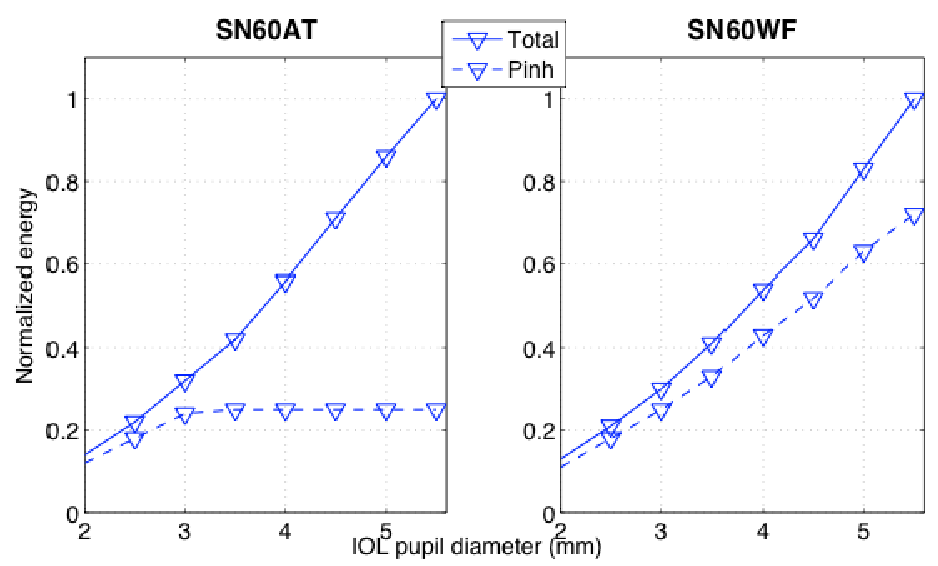

Figure 6. Energies Itotal and Ipinh measured in the image plane of monofocal IOLs as a function of the IOL illuminated diameter. Left: Spherical design. Right: Aspherical design.

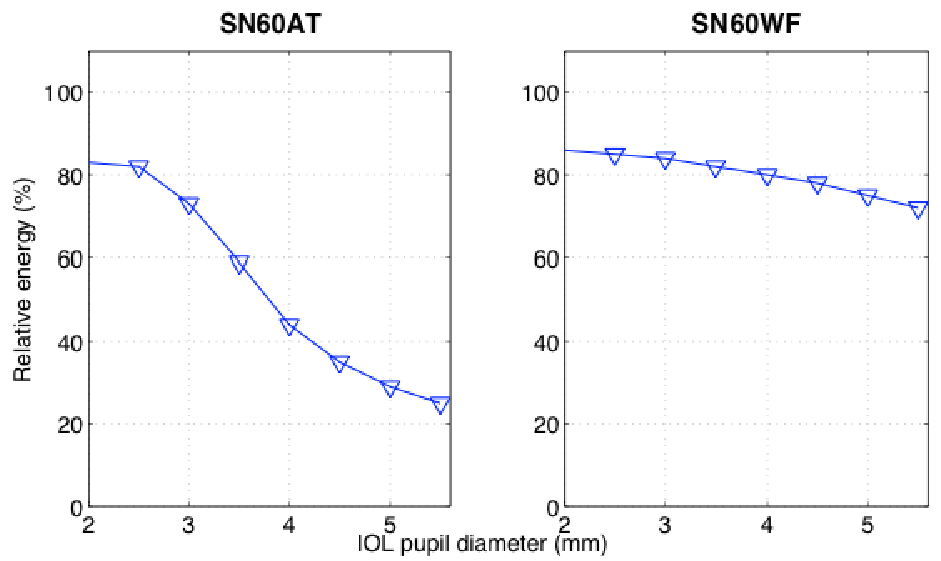

Figure 7. Energy efficiency $\left(I_{\text {pinh }} / I_{\text {total }}\right)$ as a function of the illuminated diameter of monofocal IOLs. Left: Spherical design.

Right: Aspheric design.

The results obtained with monofocal IOLs can be explained taking into account both, the level of the SA upon the IOL (Fig. 4) and the IOL design. Thus, for small IOL-pupil diameters, when the effects of the SA turn out to be low, there were little differences in the performance of the aspheric versus the spherical monofocal IOL. For larger pupils however, there are higher levels of SA upon the IOLs and the performance of the IOLs is very different depending on their design. Thus, since the spherical IOL introduces additional positive SA that adds to the one produced by the artificial cornea [24], more and more energy goes to the background for larger pupils, which strongly reduces its efficiency (Fig. 7, left). On the contrary, the aspheric IOL shows a better performance because it tends to partially compensate for the SA of the artificial cornea and manages to still focus a good amount of the additional energy in the pinhole region of the image, although it cannot impede a certain reduction of the relative energy of the image for large pupils (Fig. 7, right).

The same type of measurements was carried out in the ADMIOLs of the same add power $(+4.0 \mathrm{D})$ but different design (spherical SN60D3 vs. aspheric SN6AD3) and in ADMIOLS with the same aspheric design but different add power (+4.0 D SN6AD3 vs. +3.0 D SN6AD1). The results for the distance and near images are plotted in Fig. 8 and show that in the case of the near images there is practically no difference between the energy $I_{\text {pinh }}$ measured in any of the ADMIOLs: it increases slightly for IOL-pupil diameters up to $\sim 3.6 \mathrm{~mm}$ that corresponds to the diffractive zone of the 
IOLs and then keeps a low constant value for larger pupils. Since on the other hand, the energy $I_{\text {total }}$ of the near image increases with the EP (see Fig. 8), the energy efficiency $I_{\text {pinh }} / I_{\text {total }}$ of the near image strongly decreases for large pupils, as it is shown in Fig. 9.

These results are not unexpected taking into account that for any of these ADMIOLs the maximum aperture involved in the formation of the near image corresponds to the diffractive zone of the IOL, which is relatively small, and so is the level of SA upon the IOL (Fig. 3). Once the diffractive zone of the lens is fully illuminated, there is no way to send more energy to the near image, and as a consequence $I_{\text {pinh }}$ in the near image remains constant (Fig. 8) independently of the IOL-pupil diameter and the particular design (spherical or aspheric) or add power (+4.0D or +3.0D) of the IOL. In these conditions, the aspheric design of the SN6AD3 or the SN6AD1 proves to be no advantageous, in terms of near image energy efficiency, in front of the spherical design of the SN60D3.
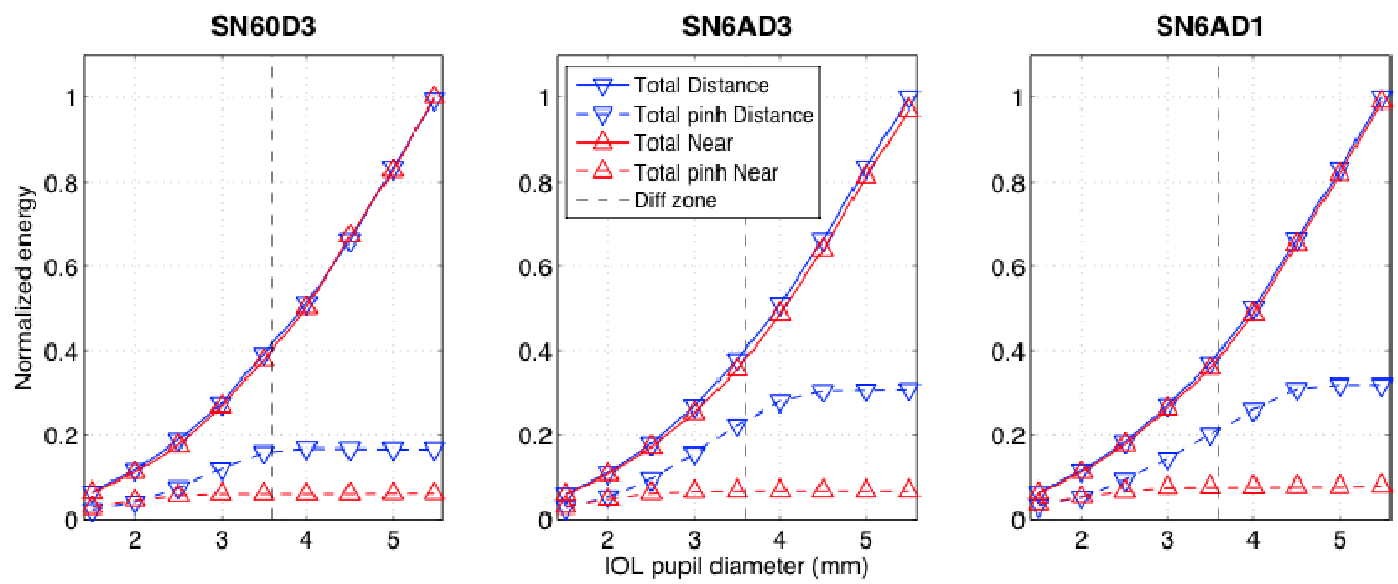

Figure 8. Energies $I_{\text {total }}$ and $I_{\text {pinh }}$ measured in the near (red lines) and distance (blue lines) image planes of the three

ADMIOL as a function of the IOL illuminated diameter.

It is in the case of the distance images and large EP diameters that the design of the ADMIOL (spherical versus aspheric) produces some differences in the experimental results. For the spherical SN60D3, as it is plotted in Fig. 8, the value of $I_{\text {pinh }}$ first increases with the EP diameter (up to 3-4 mm), and then keeps a constant value of the order of $\sim 20 \%$ for larger pupils. The results of the aspheric SN6AD3 IOL follow a similar tendency, but the measured values of $I_{\text {pinh }}$ for large pupils are about 30\% higher than the values measured for the spherical SN60D3 IOL. On the other hand, there were no significant differences in the results obtained in the ADMIOLs of aspheric design but different add power (SN6AD3 vs. SN6AD1).

SN60D3

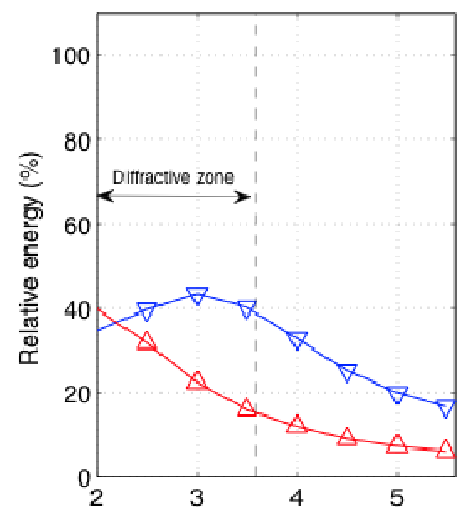

SN6AD3

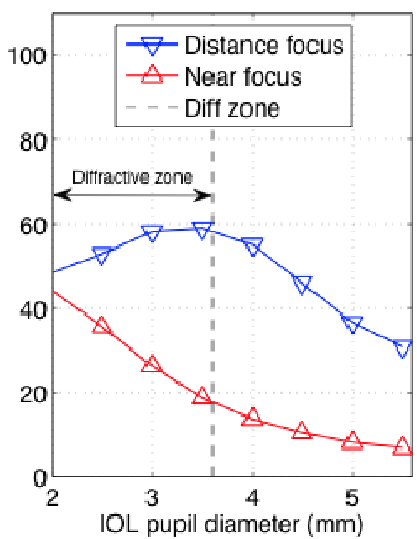

SN6AD1

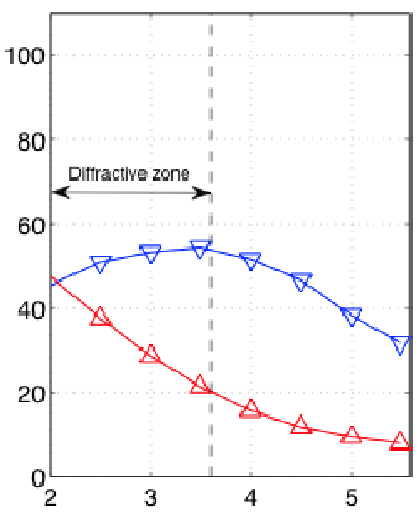

Figure 9. Energy efficiency $\left(I_{\text {pinh }} / I_{\text {total }}\right)$ as a function of the illuminated diameter of the three ADMIOLs.

Expressing these results in terms of the relative energy of the images (Fig. 9), it is seen that for the distance image both IOLs (spherical and aspheric) behave very similarly and in agreement with theoretical calculations only up to moderate 
pupil diameters (IOL-pupils of $\sim 3.6 \mathrm{~mm}$ for the spherical and $\sim 4.2 \mathrm{~mm}$ for the aspheric). In these conditions, which correspond to reduced levels of SA upon the IOL, the maximum energy efficiency achieved is around of the $40 \%$ for the spherical and $60 \%$ for the aspheric. For larger pupils when the contribution of the refractive part of the IOLs to the distance image is gaining importance, there is a clear reduction of the energy efficiency for both IOLs, in contrast with the theoretically predicted distance dominant behavior of the Acrysof ReSTOR IOLs plotted in Fig. 2. Again, the results obtained with the aspheric ADMIOLs of +4.0D and +3.0 D turn out to be quite similar

As for the distance image and large pupils, the significant differences that we have found between the experimental results and the theoretical ones put into question the theoretical distance dominant behavior of the ADMIOLs in the presence of SA. Our results show that most of the additional energy available for the distance image when the EP diameter increases, does not end up in the pinhole image but in the background. This adverse effect is even worse in the case of the spherical multifocal IOL that cannot compensate for properly the SA produced by the model eye.

\section{CONCLUSIONS}

The energy distribution of the distance and near images formed by either spherical or aspheric AMDIOLs or aspheric AMDIOLs of different add power in a model eye, has been characterized as a function of the pupil diameter. Measurements of monofocal spherical and aspheric IOLs put into evidence the influence of the level of SA in the relative energy of the images formed by the IOL. In the case of the ADMIOLs and for the distance image, the results show that with large pupils the level of SA upon the IOLs is too high to correctly focus the available energy on the image. This effect occurs even for the ADMIOLs with aspheric design (SN6AD3 and SN6AD1) and thus, in contrast with the theoretical predictions, there is a strong reduction of the relative energy of the images. In the case of the near image, similar results are obtained with all types of ADMIOLs, which is more likely due to the fact that they share the same design of the diffractive part, and the apertures involved in the near image formation are always small and so is the level of SA upon the IOL.

\section{ACKNOWLEGMENTS}

This research was supported by Spanish Ministerio de Educación y Ciencia and FEDER funds under project DPI200908879 .

\section{REFERENCES}

[1] "WHO | Priority eye diseases. http://www.who.int/blindness/causes/priority/en/index1.html."

[2] M. Larsson, C. Beckman, A. Nyström, S. Hård, and J. Sjöstrand, "Optical properties of diffractive, bifocal, intraocular lenses," Appl. Opt. 31, 2377-2384 (1992) [doi:10.1364/AO.31.002377].

[3] A. L. Cohen, "Practical design of a bifocal hologram contact lens or intraocular lens," Appl. Opt. 31, 3750-3754 (1992) [doi:10.1364/AO.31.003750].

[4] E. M. Vingolo, P. Grenga, L. Iacobelli, and R. Grenga, "Visual acuity and contrast sensitivity: AcrySof ReSTOR apodized diffractive versus AcrySof SA60AT monofocal intraocular lenses," Journal of Cataract \& Refractive Surgery 33, 1244-1247 (2007) [doi:10.1016/j.jcrs.2007.03.052].

[5] G. Schmidinger, C. Simader, I. Dejacoruhswurm, C. Skorpik, and S. Pieh, "Contrast sensitivity function in eyes with diffractive bifocal intraocular lenses," Journal of Cataract \& Refractive Surgery 31, 2076-2083 (2005) [doi:10.1016/j.jcrs.2005.04.037].

[6] F. Alba-Bueno, F. Vega, and M. S. Millán, "Design of a Test Bench for Intraocular Lens Optical Characterization,” J. Phys.: Conf. Ser. 274, 012105 (2011).

[7] T. Eppig, K. Scholz, and A. Langenbucher, "Assessing the optical performance of multifocal (diffractive) intraocular lenses," Ophthalmic Physiol Opt 28, 467-474 (2008).

[8] S. Pieh, W. Fiala, A. Malz, and W. Stork, "In Vitro Strehl Ratios with Spherical, Aberration-Free, Average, and Customized Spherical Aberration-Correcting Intraocular Lenses," Investigative Ophthalmology \& Visual Science 50, $1264-1270$ (2009).

[9] S. Pieh, P. Marvan, B. Lackner, G. Hanselmayer, G. Schmidinger, R. Leitgeb, M. Sticker, C. K. Hitzenberger, A. F. Fercher, et al., "Quantitative Performance of Bifocal and Multifocal Intraocular Lenses in a Model Eye: Point Spread Function in Multifocal Intraocular Lenses," Arch Ophthalmol 120, $23-28$ (2002). 
[10] T. Terwee, H. Weeber, M. van der Mooren, and P. Piers, "Visualization of the retinal image in an eye model with spherical and aspheric, diffractive, and refractive multifocal intraocular lenses," J Refract Surg 24, 223-232 (2008).

[11] N. E. de Vries, C. A. B. Webers, R. Montés-Micó, T. Ferrer-Blasco, and R. M. M. A. Nuijts, "Visual outcomes after cataract surgery with implantation of a $+3.00 \mathrm{D}$ or $+4.00 \mathrm{D}$ aspheric diffractive multifocal intraocular lens: Comparative study," J Cataract Refract Surg 36, 1316-1322 (2010).

[12] N. E. de Vries, C. A. B. Webers, F. Verbakel, J. de Brabander, T. T. Berendschot, Y. Y. Y. Cheng, M. Doors, and R. M. M. A. Nuijts, "Visual outcome and patient satisfaction after multifocal intraocular lens implantation: aspheric versus spherical design," J Cataract Refract Surg 36, 1897-1904 (2010),

[13] T. Kohnen, R. Nuijts, P. Levy, E. Haefliger, and J. F. Alfonso, "Visual function after bilateral implantation of apodized diffractive aspheric multifocal intraocular lenses with a +3.0 D addition," J Cataract Refract Surg 35, 2062-2069 (2009).

[14] G. Munoz, C. Albarrandiego, R. Montesmico, A. Rodriguezgalietero, and J. Alio, "Spherical aberration and contrast sensitivity after cataract surgery with the Tecnis Z9000 intraocular lens," J Cataract Refract Surg 32, 1320-1327 (2006).

[15] J. A. Davison and M. J. Simpson, "How does the ReSTOR lens work?," Review of Refractive Surgery, 18-20 (2004).

[16] J. A. Davison and M. J. Simpson, "History and development of the apodized diffractive intraocular lens," J Cataract Refract Surg 32, 849-858 (2006).

[17] A. Guirao, M. Redondo, and P. Artal, "Optical aberrations of the human cornea as a function of age," J Opt Soc Am A Opt Image Sci Vis 17, 1697-1702 (2000).

[18] L. Wang, E. Dai, D. D. Koch, and A. Nathoo, “Optical aberrations of the human anterior cornea," J Cataract Refract Surg 29, 1514-1521 (2003).

[19] J. Schwiegerling, "Intraocular lenses," in Handbook of optics: Vision and Vision Optics, M. Bass, J. M. Enoch, V. Lakshminarayanan, V. N. Mahajan, and E. V. Stryland, Eds., McGraw Hill Professional, New York (2009).

[20] C. Lee and J. Simpson, "Diffractive multifocal ophthalmic lens," U.S. Patent No. 5699142 (1997).

[21] A. L. Cohen, "Diffractive bifocal lens designs," Optom Vis Sci 70, 461-468 (1993).

[22] N. E. de Vries, L. Franssen, C. A. B. Webers, N. G. Tahzib, Y. Y. Y. Cheng, F. Hendrikse, K. F. Tjia, T. J. T. P. van den Berg, and R. M. M. A. Nuijts, "Intraocular straylight after implantation of the multifocal AcrySof ReSTOR SA60D3 diffractive intraocular lens," J Cataract Refract Surg 34, 957-962 (2008).

[23] S. Norrby, P. Piers, C. Campbell, and M. van der Mooren, "Model eyes for evaluation of intraocular lenses," Appl Opt 46, 6595-6605 (2007).

[24] M. Packer, I. H. Fine, and R. S. Hoffman, "Aspheric intraocular lens selection: the evolution of refractive cataract surgery," Curr Opin Ophthalmol 19, 1-4 (2008).

[25] International Organization for Standarization, "Ophthalmic Implants, Intraocular lenses Part 2: Optical Properties and Test Methods."

[26] F. Vega, M. S. Millán, and B. Wells, "Spherical lens versus aspheric artificial cornea for intraocular lens testing," Opt. Lett. 35, 1539-1541 (2010).

[27] W.K. Pratt, "Digital Image Processing”, 2nd ed., Wiley, New-York, 1991. 